\title{
AN APPROXIMATION PROBLEM WITH A NEW CHARACTERISTIC AND A PROBLEM ON MEAN APPROXIMATION ON ARCS IN A COMPLEX PLANE
}

\author{
JAMAL MAMEDKHANOV AND IRADA DADASHOVA
}

\begin{abstract}
In the paper classical approximation theorems are investigated on the curves in a complex domain. In particular, direct and inverse theorems are cited on the curves $\Gamma$ in a complex domain in the metric $L_{p}(\Gamma)$. The obtained results remain new on the segment $[-1,1]$ as well. Furthermore, a new characteristic by which the author obtained the analogies of MarkovBernstein type and also S. M. Nikolskiy type classical estimates is also considered.

In future, it is presupposed to get the analogies of classic theorems of Jackson, JacksonBernstein, Bernstein and Nikolskiy-Timan-Dzyadyk by these characteristics.
\end{abstract}

Mathematics subject classification (2010): 30E10, 41A10, 41A17.

Keywords and phrases: Polynomial approximation, classical approximation theorems, constructive characteristics, quasiconformal curves.

\section{REFERENCES}

[1] V. V. ANDRIEVSKII, Polynomial approximation of analytic functions on a finite number of continua in the complex plane, J. Approx. Theory 133 (2005), 238-244.

[2] V. I. BELYI, Conformal mapping and approximation of analytic functions in quasiconformal boundary domains, (Russian), Math.Sb. 102 (144), 3 (1977), 331-361.

[3] Z. Ditzian And V. Totik, Moduli of Smoothness, Springer Series in Computational Mathematics 9, Springer-Verlag, New York, 1987.

[4] V. K. DZYADYK, Introduction to the theory of approximation of functions by polynomials, (Russian), 'Nauka' M., 1977.

[5] V. K. DZyAdy K AND G. A. AliBeKov, Uniform approximation of functions of a complex variable on closed sets with corners, Mat.Sb. 75(117), 4 (1968), 502-557.

[6] G. David, L'integrale de Cauchy sur le corbes rectifiables, Prepublications Univ. Paris-Sud., Dept. Math., 82 TOS, (1982), 1-21.

[7] E. P. Dolzhenko And V. I. Danchenko, Mapping of sets of finite $\alpha$-measure by rational functions, (Russian), Math. USSR Izv. 51, 6 (1987), 1309-1321.

[8] B. V. KhVEDELIDze, The method of Cauchy type integrals in discontinuous boundary value problems, Modern problems of mathematics, M.: Itogi 7 (1975), 5-163.

[9] J. I. MAMEDKhanov, On the problems of J. Walsh and D. Newman, associated with the polynomial approximation, (Russian), Abstracts of papers of All-Union Symposium on Theory of approximation of functions in the complex domain, UFA, 1980.

[10] J. I. MAMEDKHANOv, On the arcs with the property of Jackson, (Russian), Abstracts of International Colloquium on Fourier analysis, Budapest, 1976.

[11] J. I. Mamedkhanov, Globally local approximation on arcs, Trans. Acad. Sci. Azerb., Ser. Phys.Tech. Math. Sci. 20, 1 (2000), 60-67.

[12] J. I. Mamedkhanov, On a problem of J. Newman, Abstracts of International conference on approximation theory, (Russian), Koluga, 1975.

[13] V. V. MAimeSKul, The J-property of Jordan arcs, (Russian), Ukrain. Mat. Zh. 40, 5 (1988), 647649. 
[14] J. I. Mamedkhanov And I. B. Dadashova, S.N.Bernstein Type Estimations in the Mean on the Curves in a Complex Plane, Abstract And Applied Analysis 2009 (2009), 19 pages. doi:10.1155/2009/165194.

[15] J. I. Mamedkhanov, On S. M. Nikolsky type inequalities with a new characteristic, (Russian), Doklady Mathematics 435, 2 (2010), 12.

[16] J. I. Mamed Khanov, Problems on best polynomial approximation in the complex plane, Theory of functions and approximations, Part 1, Saratov. Gos. Univ., Saratov, 1982, 149-156.

[17] D. J. Newman, Jackson's theorem on complex arcs, J. Approximation Theory 10, 3 (1974), 206-217.

[18] I. I. PRIVALOV, Boundary properties of single-valued analytic functions, (Russian), "Nauka" M., 1950.

[19] Ch. Pommerenke, Boundary Behavior of Conformal Maps, Grundlehren der mathematischen Wissenschaften, Volume 299, 1992, 300 pp.

[20] M. K. Potapov, Some questions of best approximation in the metric $L_{p}$, Thesis, Moscow, 1956.

[21] V. V. SAlayev, Primal and inverse estimations for Cauchy's singular integral on a closed curve, (Russian), Math. Zametki 19 (1976), 365-380.

[22] S. B. STECHKIN, On the best approximation order of continuous functions, (Russian), Izv. AN SSSR, ser.mat. 15, 3 (1951), 219-242.

[23] J. L. WALSH, Interpolation and approximation by rational functions in the complex domain, (Russian), IL, M., 1961. 